\title{
AC 2012-4405: ECE CURRICULUM IMPROVEMENT TO INCORPORATE GLOBAL LEARNING
}

\section{Dr. Phillip A. Mlsna, Northern Arizona University}

Phillip Mlsna is currently an Associate Professor in the Electrical Engineering and Computer Science Department at Northern Arizona University. His primary research interests are in image processing, computer vision, engineering education, and academic ethics. He has extensive experience as a Computer Hardware Engineer at Hewlett-Packard.

\section{Dr. Niranjan Venkatraman, Northern Arizona University}

Niranjan Venkatraman obtained his Ph.D. in electrical and computer engineering from the University of Florida, Gainesville, in 2004, and is currently an Associate Professor in the Department of Electrical Engineering and Computer Science at Northern Arizona University, Flagstaff, Ariz.. His areas of interest are systems and control, global learning, and engineering pedagogy.

Dr. Sheryl L. Howard, Northern Arizona University 


\section{ECE Curriculum Improvement to Incorporate Global Learning}

\section{I. introduction}

Traditional Electrical Engineering curricula include courses in the fundamentals of electrical engineering, senior elective courses in specialized topics, and a capstone senior project. Labs provide students with experience in hardware and software design, and the capstone project ideally allows them to take an electrical engineering project from inception to completion. Material about cultural diversity and global engagement was considered the realm of Liberal Studies, and, if not irrelevant to engineering, of minor importance.

In the past decade, recognition of the importance of global learning, which encompasses cultural diversity and global engagement, to the fields of engineering has grown significantly ${ }^{1}$. We live in a global economy. Today's engineer is a global engineer; they will work for companies that have a global presence and may work overseas. Today's engineer may work on projects whose teams span the globe, and will most likely have clients in multiple countries. Therefore, an understanding of and appreciation for cultural diversity and the fact that we work in a global marketplace is critical for our graduating students to succeed as electrical engineers ${ }^{7 ; 8 ; 10}$.

In recognition of the importance of global learning and the need to present its relevance to our engineering students, the authors, with the support of faculty in our Electrical and Computer Engineering programs, embarked on a plan to incorporate global learning directly into our ECE curriculum. This was done as part of a university-wide initiative to include global learning in department curricula. We viewed this initiative as a great opportunity to align our ECE program curricula closer to the needs of employers and future engineers participating in a global economy. Students who have a broader view of their engineering education, and understand how engineering solutions impact the environment and cultures, as well as how culture impacts engineering, are better prepared for their career as an engineer in a global society.

Three Global Learning Outcomes were identified as part of the university-wide initiative: 1) Global Engagement; 2) Diversity Education; and 3) Environmental Sustainability. We agree with this division of global learning aspects. While sustainability may not initially seem to be a component of global learning, it is an aspect that is truly global in its scope, and of concern to all peoples on this Earth. Disregard for the importance of sustainability has global effects. Furthermore, sustainability is a key feature of good engineering design and comprises much more than recyclability or use of green power, although these are important aspects. Efficiency in design and implementation is simply good engineering practice. Wasting resources of any kind is inefficient design and costly for the manufacturer. Sustainability, when viewed as efficient design, is a key component of our engineering curriculum ${ }^{9}$.

This paper presents both the process and results of incorporating these three global learning components into our Electrial and Computer Engineering curriculum. As a result of this work, we can directly assess how well our students are integrating the global learning outcomes as they advance through the Electrical Engineering or Computer Engineering programs. Incorporation of global learning into our curriculum gives us an opportunity to better prepare our students for careers as 
truly global engineers ${ }^{3 ; 6}$.

Three key results of this integration of global learning elements into the ECE curriculum are the following:

1. Several elements of global learning were already present in our ECE curriculum, but were not formalized or assessed. The most common were:

(a) Discussion of historical background of course topic;

(b) Sustainability, in the form of efficient design or use of resources (for example, minimized logic, efficient code or spectrum usage).

2. Global learning assessment supports and strengthens assessment of ABET Criterion 3 outcomes $\mathrm{a}, \mathrm{h}$ and $\mathrm{j}$ in particular ${ }^{2}$.

3. Incorporation of global learning into ECE curriculum can be accomplished with little additional faculty workload, recognizing that global learning assessment also serves to assess some ABET outcomes.

Additionally, alumni from industry and regional employers expressed strong support of our efforts to incorporate global learning into our ECE curriculum. They related several instances where a lack of cultural understanding caused problems and/or delays with their global clients, subcontractors and employees.

The process we followed while incorporating global learning into the ECE curriculum is described below in Section II.

\section{II. process for incorporating global learning}

Faculty buy-in of the importance of global learning to ECE, and support for its incorporation into our ECE curriculum, was critical. Thus our initial action was to discuss global learning and the advantages of its incorporation into our ECE curriculum with department faculty. With department approval, we then began the process of integrating global learning aspects into our curriculum, and developing methodologies to assess them. A key goal was to include global learning components that would also support ABET and program outcomes. Assessment thus serves multiple outcomes; addition of global learning aspects and assessment has minimal impact on faculty load. Another goal was to utilize existing global learning efforts which were not currently recognized as such or being assessed. Again, this minimizes faculty workload by utilizing what they are already doing.

The first step in incorporating global learning into our ECE curriculum was to evaluate our curriculum and determine what, if any, global learning aspects were already present. This required input from department faculty indicating course topics in sustainability, diversity and global engagement which might not be formalized or assessed, but were discussed in their courses, even if on an ad hoc basis. Our evaluation of existing global learning elements in our curriculum is discussed in detail in Section III. After determining the global learning aspects already present in our curriculum, we categorized those aspects into each of the three global learning outcomes 
of sustainability, diversity and global engagement. From there, we could see which global learning outcomes were already well-supported in our curriculum, and only needed formalization and assessment, and which outcomes were only weakly supported, needing development of further efforts to strengthen that global learning outcome in our curriculum. To minimize additional faculty workload, the aspects that would support the particular global learning outcome should also support our ABET and program outcomes whenever possible, so that assessment can target multiple outcomes. One important aspect proposed was a new multidisciplinary course presenting key global contributions of engineering from several cultures throughout history. These new efforts are described in Section IV. In Section V, we describe a new course that is designed to directly address multiple aspects of global learning in our engineering curriculum.

Next, we examined our ECE program outcomes to determine how effectively they addressed the three global learning outcomes of sustainability, diversity and global engagement. We added one new program outcome and slightly modified four other program outcomes to better support the global learning outcomes. These modified program outcomes are presented in Section VI, with the global learning outcomes they address.

Our ABET and program outcomes were evaluated with respect to the global learning outcomes, to determine a mapping between global learning and ABET or program outcomes. Each global learning outcome was supported by one or more ABET Criterion 3 outcomes. Modifying our ECE program outcomes to include all three global learning components strengthened the relationship between our ECE program outcomes and ABET outcomes, with better support for several ABET outcomes. The mapping between our modified program outcomes, including global learning components, and the ABET outcomes is presented in Section VII.

The next step was to develop or modify existing assessment methodologies for our ECE program outcomes which would support assessment of the global learning outcomes. As a crucial last step, faculty support of the modified program outcomes and their assessment methodologies was obtained. The new or modified assessment methodologies are presented in Section VIII. Conclusions based on our discoveries during the process of integrating global learning elements into our ECE curriculum are presented in Section IX.

\section{III. existing curricular efforts supporting global learning outcomes}

As part of our analysis, we examined the ECE program to determine what curricular efforts, if any, already addressed global, sustainability or diversity aspects. As noted below, many of these existing efforts are not currently being assessed. Several of these aspects will be specifically included in assessment, to evaluate their corresponding Global Learning outcome, the appropriate ECE program outcome and ABET objective(s).

\section{Global Engagement}

(a) Contemporary issues and the impact of electrical engineering on global society: Engineering Design (required course). Students present a slideshow as a group on teamselected contemporary ethical topics in electrical engineering. Current assessment efforts in this course evaluate regional ethical concerns, with no mention on global ethics. 
(b) Historical perspective and biographical information on inventors and scientists: presented briefly in many courses, including Digital Logic, Basic Electrical Engineering, Microprocessors, Engineering Mathematics, Signals and Systems (all required) and Communication Systems (elective). No current assessment efforts.

\section{Environmental Sustainability}

(a) Electromagnetics (required course) Junkyard Generator Lab: students build generator out of "found" or recycled items. Labs are graded each year but no assessment evaluation is performed.

(b) Sustainable methods of power generation discussed in Engineering Design and Power Systems (elective). No ongoing assessment with respect to sustainability.

(c) Ethical concerns in electrical engineering: issues which may impact the environment, such as power generation and semiconductor fabrication, are discussed in Engineering Design. Assessment of an ethics paper is ongoing for ABET accreditation.

(d) Efficiency via design minimization: Ongoing assessment with K-map problem on Digital Logic final exam and assessment quiz in Microprocessors.

(e) Spectral efficiency, maximizing use of limited spectral resources: presented in Signals and Systems and emphasized in Communication Systems. No ongoing assessment.

\section{Diversity Education}

(a) The ECE program has both a diverse student population and diverse faculty. Assessment of student population breakdown by gender, ethnicity and nationality is ongoing for ABET accreditation.

(b) Several international exchange students take classes in our ECE program each year. Most of our exchange students are from European and Middle Eastern countries. No current assessment or enumeration of exchange students is currently being done at the ECE program level.

From the list shown above, we observe that, while each Global Learning Outcome has some curricular resources and efforts that already support that outcome, very few of these efforts presented above are currently being assessed. To implement global learning more fully in the ECE program as well as improve evaluation of related ABET and program outcomes, assessment methodologies for important efforts will be developed. We also note that Global Engagement and Diversity Education have only two existing curricular efforts listed under each outcome.

\section{IV. future curricular efforts supporting global learning outcomes}

Future efforts to enhance inclusion of each Global Learning Outcome, especially Global Engagement and Diversity Education, in the ECE program and improve assessment are outlined below.

\section{Global Engagement}

(a) Development of a new multidisciplinary course that presents the global contributions of engineering by many different cultures over time. This course could be a liberal studies course or seminar, and would be taught at a level accessible to all engineering, and probably all science, students. 
(b) A 4-year ECE program that allows study abroad without delaying graduation: a 6or 8-week summer semester abroad for engineering students between freshman and sophomore year is being trialed next summer. Two courses required for most engineering disciplines are offered in this summer study abroad engineering program. The ECE program encourages student participation in this study abroad program.

(c) Assessment of historical perspective presented in selected courses.

(d) Assessment of impact of ECE on global society in Engineering Design.

\section{Environmental Sustainability}

(a) Develop clearly-targeted assessment methods for this Global Learning Outcome that also support the modified program outcome on sustainability.

(b) Increase student awareness of Intro to Environmental Engineering and Environmental Sustainability as possible Science Elective choices in their ECE program. Each ECE student must take two Science Elective courses.

\section{Diversity Education}

(a) Development of new multidisciplinary course previously discussed also supports this outcome.

(b) Potential inclusion of material on differing engineering solutions for different cultures, due to varied cultural/environmental/economic constraints. One of the engineering design courses would be best for this topic. Assessment of student understanding of the need for engineering solutions that consider cultural, environmental and economic requirements would be developed if this material is presented.

(c) Gather data on number of exchange students per year taking ECE classes. Potentially develop survey for exchange student to assess their educational experience with respect to the ECE program.

\section{V. a new engineering course}

To present key global contributions of engineering throughout history, and strengthen both global engagement and diversity components of global learning in our ECE curriculum, we have proposed a new freshman course tentatively called "Engineering and Civilization". The purpose of this course is to provide students with perspective and appreciation for the historical and cultural contexts in which key technologies were developed. Emphasis will also be placed on societal and environmental impacts, both positive and negative. Students will be expected to be able to explain these developments and their impacts from multiple viewpoints such as the nature of the technical progress, the changes in the society that produced it, the effect on the subsequent careers and fortunes of its developers, and its importance and usefulness in today's engineering practice.

This new course is envisioned as based upon work done at Rutgers University and the University of California at Merced in cooperation with the IEEE Global History Network ${ }^{4}$ and the Society for the History of Technology 5 . Their courses have been built on the concept of covering ten to fifteen key technological developments structured as learning modules. The focus will not be on strictly covering the historical flow of technological development per se, but rather using selected major topics from technological history as springboards from which to examine cultural context, societal and personal impacts, the enabling of subsequent advances, and even ethical issues. Some 
examples of key technological advances we are considering as module topics include the sailing ship, the steam engine, computers, gunpowder, powered flight, radio communication, skyscrapers, and refrigeration. By including several topics of a contemporary nature, such as nanotechnology or the internet, the course will help students better understand some of the current trends and emerging technologies that are likely to affect their own careers. We believe it is important for students to understand how technological progress actually occurs in order for them to better prepare to participate in this process.

\section{VI. overview of ECE program outcomes and proposed changes}

The ECE program outcomes were analyzed with respect to their effectiveness in addressing the Global Learning Outcomes. The ECE program outcomes are listed as follows.

1.1 - Be a leader in educational innovation and the use of technology in providing a quality educational experience.

1.2 - Attract and retain well-qualified students.

1.3 - Foster advising and mentoring relationships between faculty, industry and students.

2.1 - Possess professional skills and knowledge of the design process.

2.2 - Ability to function in disciplinary and multi-disciplinary teams.

2.3 - Possess abilities to effectively communicate orally.

2.4 - Possess abilities to effectively communicate in writing.

2.5 - Abilities in creativity, critical thinking and problem identification, formulation and solving.

3.1 - Ability to apply knowledge of physics and mathematics (including calculus, linear algebra, complex variables and differential equations).

3.2 - Ability to apply knowledge of probability, statistics, Laplace transforms and Fourier transforms.

3.3 - Ability to design and conduct scientific and engineering experiments.

3.4 - Motivation and skills needed for lifelong learning.

3.5 - Ability to use industry standard analysis and design tools.

4.1 - Ability to relate a broad education and contemporary issues to engineering solutions and their impact in a societal and global context.

4.2 - An appreciation and understanding of professional and ethical responsibility.

4.3 - Attract and retain under-represented students.

Our analysis of the above outcomes showed they were clearly lacking in the Global Learning Outcomes. In order to address these concerns, we modified some of the existing outcomes and added one new outcome to the above list. The modified/new outcomes are listed below, with changes in bold; the Global Learning Outcome(s) covered by each program outcome are clearly shown at the start of the program outcome statement.

\section{4 - (GLOBAL, DIVERSITY) An appreciation and understanding of the global and diverse nature of science and engineering.}

2.1 - (SUSTAINABILITY) Possess professional skills and knowledge of the engineering design process, with an emphasis on sustainability.

2.2 - (DIVERSITY) Ability to function in disciplinary,andmulti-disciplinary and diverse teams. 
4.1 - (GLOBAL) Ability to relate a broad education and contemporary issues to engineering solutions and their impact in a societal and global context.

4.2 - (GLOBAL, SUSTAINABILITY) An appreciation and understanding of professional and ethical responsibility in a sustainable, societal and global context.

4.3 - (DIVERSITY)Attract and retain a diverse group of under-represented students.

Table $\mathrm{V}$ is a significant table which shows the achievement levels of the various program outcomes assessed to date. The new or modified outcomes have not been assessed fully as yet, so their achievements are listed as uncertain, or in some cases, adequate. The outcomes related to the Global Learning Outcomes are shaded in grey in the below table.

Table V Current Achievements of ECE Program Outcomes

\begin{tabular}{|c|c|c|c|c|c|c|c|c|c|c|c|c|c|c|c|c|c|}
\hline & \multicolumn{10}{|c|}{ Electrical Engineering Program Outcomes } \\
\hline Achievement & 1.1 & 1.2 & 1.3 & 1.4 & 2.1 & 2.2 & 2.3 & 2.4 & 2.5 & 3.1 & 3.2 & 3.3 & 3.4 & 3.5 & 4.1 & 4.2 & 4.3 \\
\hline High & $\bullet$ & $\bullet$ & $\bullet$ & & & & & & & & $\bullet$ & & & & & & \\
\hline Good & & & & & & & $\bullet$ & $\bullet$ & $\bullet$ & $\bullet$ & & $\bullet$ & $\bullet$ & $\bullet$ & & & \\
\hline Adequate & & & & & $\bullet$ & $\bullet$ & & & & & & & & & $\bullet$ & $\bullet$ & $\bullet$ \\
\hline Uncertain & & & & $\bullet$ & & & & & & & & & & & & & \\
\hline
\end{tabular}

\section{VII. relationship between program outcomes and ABET outcomes}

The relationship between the Electrical Engineering program outcomes at our university is a fairly direct mapping of the ABET (a) through (k) outcomes. Table VI displays the mapping showing the direct and lesser dependencies that we feel exist between the two sets of outcomes. For purposes of compactness, the ECE outcomes are indicated by their numbers and the ABET outcomes are referred to by their familiar letter designation. A full circle indicates a direct relationship between the outcomes, while a half circle indicates a lesser relationship.

The ABET outcomes are listed below, with outcomes related to Global Learning in bold:

(a) an ability to apply knowledge of mathematics, science, and engineering

(b) an ability to design and conduct experiments, as well as to analyze and interpret data

(c) an ability to design a system, component, or process to meet desired needs within realistic constraints such as economic, environmental, social, political, ethical, health and safety, manufacturability, and sustainability

(d) an ability to function on multidisciplinary teams

(e) an ability to identify, formulate, and solve engineering problems

(f) an understanding of professional and ethical responsibility

(g) an ability to communicate effectively

(h) the broad education necessary to understand the impact of engineering solutions in a global, economic, environmental, and societal context

(i) a recognition of the need for, and an ability to engage in life-long learning

(j) a knowledge of contemporary issues

(k) an ability to use the techniques, skills, and modern engineering tools necessary for engineering practice

The following Table VI shows only the mapping of the new or modified outcomes to the ABET criteria. 
Table VI Mapping of New or Modified ECE Program Outcomes and ABET Outcomes

\begin{tabular}{|l|c|c|c|c|c|c|c|c|c|c|c|}
\hline & \multicolumn{5}{|c|}{ ABET Criterion 3 Outcomes } \\
\hline ECE Program Outcomes & $\mathbf{a}$ & $\mathbf{b}$ & $\mathbf{c}$ & $\mathbf{d}$ & $\mathbf{e}$ & $\mathbf{f}$ & $\mathbf{g}$ & $\mathbf{h}$ & $\mathbf{i}$ & $\mathbf{j}$ & $\mathbf{k}$ \\
\hline 1.4 Understanding of global and diverse nature & & & & $\mathbf{D}$ & & & & $\mathbf{D}$ & & $\mathbf{D}$ & \\
\hline 2.1 Professional skills, sustainable design process & & $\mathbf{D}$ & $\mathbf{0}$ & & & & & & $\mathbf{D}$ & & \\
\hline 2.2 Disciplinary, multi-disciplinary and diverse teams & & & & $\mathbf{O}$ & & & & & & & \\
\hline 4.1 Broad education, contemporary issues and impact on society & & & $\mathbf{D}$ & & & & & $\mathbf{0}$ & & $\mathbf{0}$ & \\
\hline 4.2 Professional and ethical responsibility in global context & & & $\mathbf{D}$ & & & $\mathbf{0}$ & & & & & \\
\hline 4.3 Attract and retain under-represented students & & & & $\mathbf{D}$ & & & & & & & \\
\hline
\end{tabular}

\section{VIII. assessment of new and modified ECE program outcomes}

This section discusses the assessment process and methodology to be used for each new or modified ECE program outcome. Assessment of the outcomes below also directly leads to assessment of the global learning outcomes as indicated, and to the ABET criteria according to the mapping in Table VI. This assessment is primarily inspired by the university's Global Learning Initiative and is therefore currently aimed at measuring the degree of satisfaction of this initiative's goals. However, the authors envision and are currently planning a broader assessment scope to measure the effect of such global perspective training on the subsequent career paths of students. By tracking and surveying students for several years after graduation, it should be practical to apply this information to refine key curriculum aspects regarding global learning as well as other areas.

Outcome 1.4: (GLOBAL, DIVERSITY) An appreciation and understanding of the global and diverse nature of the origins and development of science and engineering.

a. Process - This outcome will be assessed by evaluating student essays in the proposed new course on Historical Perspectives in Engineering. The essays will ask the students to describe their understanding of the global nature of the development of the engineering disciplines, as well as the diverse nature of the development. The essays will be assessed by three faculty members by using a rubric which will be generated for the purpose.

b. Changes to be adopted - A major developmental change will be the introduction of a freshman/sophomore seminar course, which introduces the students to the historical development of the engineering disciplines. This course will be mandatory for all incoming ECE freshmen.

Outcome 2.1: (SUSTAINABILITY) Possess professional skills and knowledge of the engineering design process, with an emphasis on sustainability.

a. Process - This outcome is assessed by evaluating student reports and certain topical essays in the Engineering Design sequence courses. The reports detail the students' perception of the design process, as well as sustainable engineering concepts applied to the process.

b. Changes to be adopted - A topic on the Sustainable Design process will be included. Student reports will contain a clear section explaining how sustainable engineering guidelines were used in the design phase of their projects.

Outcome 2.2: (DIVERSITY) Ability to function in disciplinary, multi-disciplinary and diverse teams.

a. Process - Current assessment evaluates group presentations on student capstone projects. Teams are typically disciplinary, but a few teams are multi-disciplinary, usually including Mechanical as well as ECE students. A previously-developed rubric, which covers many aspects of the team 
presentation, is used to evaluate the students with respect to this outcome for one category, "Team member interaction".

b. Changes to be adopted - The current rubric category does not include diversity. A question on diversity, while not applicable to the rubric, would be an appropriate topic for a class survey in Engineering Design and Senior Design. A survey question or questions could ask the student to i) evaluate the diversity of their own capstone team; ii) evaluate the diversity of the class as a whole; iii) evaluate what they learned as a result of a diverse class population. Data can also be collected by the course instructor as to the composition and diversity of each year's course.

Outcome 4.1: (GLOBAL) Ability to relate a broad education and contemporary issues to engineering solutions and their impact in a societal and global context.

a. Process - This outcome is analyzed based on twenty-minute team presentations of electrical engineering contemporary issues given in Engineering Design. A developed rubric is used to assess the presentations. Both an alumni survey question and graduating senior survey question were also used to assess this outcome. b. Changes to be adopted - Incorporation of contemporary engineering issues that clearly have a global and societal impact into the contemporary issues module of Engineering Design would be an ideal method to address this outcome.

Outcome 4.2: (GLOBAL, SUSTAINABILITY) An appreciation and understanding of professional and ethical responsibility in a sustainable, societal and global context.

a. Process - Student performance on this outcome will be analyzed by three Electrical Engineering faculty members from Engineering Design individually-prepared ethics papers using a rubric developed by the ECE faculty for this purpose. An individually prepared ethics paper in Senior Design will also be used to assess ethics as related to sustainable engineering design. In addition, graduating seniors and alumni who graduated since 2000 will be asked to rate how well their university education assisted them in developing their understanding of global professional and ethical responsibility through a survey.

b. Changes to be adopted - The students in the Electrical Engineering program are required take a course on Ethics early in their program. In their junior year, the students are required to take Engineering Design. It is proposed that the instructor for this course build on the student learning in Ethics, and include lecture material on global ethics - how ethical perception varies in a global and societal context. Further, in Senior Design, it is proposed that the students write an essay on ethics related to sustainable engineering design. The current rubric will be redesigned for this purpose.

\section{Outcome 4.3: (DIVERSITY) Attract and retain a diverse group of under-represented stu-} dents.

a. Process - Data on the number of incoming under-represented students in ECE, as well as graduating under-represented ECE students, and yearly retention data for ECE students, categorized by gender and ethnicity, is collected from the Planning and Institutional Research department. Although the number of under-represented students is small, overall trends can be evaluated on a yearly basis using under-represented students in the entire ECE program. These numbers are also compared to the average university-wide percentage of incoming under-represented students, as well as retention and graduation rates.

b. Changes to be adopted - Increase targeted efforts to recruit under-represented students with a potential interest in ECE or STEM fields in general. Collect and analyze data on scholarships 
and internships obtained by, as well as hiring and faculty support of, under-represented students in ECE. Increase student awareness of scholarship, internship and research opportunities with inclass discussion.

\section{IX. conclusions}

This paper presented our efforts to incorporate global learning into the ECE curriculum. We discovered existing curricular efforts that already support the Global Learning Outcomes, and examined existing ECE program outcomes with respect to global learning aspects. Some global learning efforts existed in our ECE curriculum that had not been previously recognized or assessed. Several courses presented some historical background for various topics and biographical information on key engineers, scientists and mathematicians. This was done on an ad hoc basis, as time permitted, and was not assessed. Sustainability, in the form of efficient design or use of resources, is a common thread throughout most of our ECE courses; however, it was rarely assessed and was not part of the existing program outcomes.

Shortcomings in curricular efforts as well as existing program outcomes were discovered. Modifications were made where appropriate to incorporate all three Global Learning Outcomes into the ECE program. A mapping of our ECE program outcomes to the ABET Criterion 3 outcomes was similarly modified to include the new and updated ECE program outcomes, resulting in better coverage of several of the ABET outcomes. Previous development of this outcome mapping was key to determining which program outcomes best supported ABET outcomes with global learning aspects.

Assessment of student realization of the Global Learning Outcomes is a critical part of implementing global learning into our ECE program. As part of our ABET accreditation process, we had already developed assessment methodologies and tools for each of our ECE program outcomes and educational objectives. Enhancement of our existing assessment methodologies to support the modified program outcomes that support the Global Learning Outcomes was relatively straightforward. Assessment of the Global Learning Objectives can also directly assess these ABET objectives relating to diversity, sustainability and global engagement. Incorporation of global learning into the ECE curricular process also strengthens the ECE program in its accreditation process. The enhanced assessment of the Global Learning Outcomes translates directly to better, more thorough, assessment of our ABET outcomes for accreditation purposes.

All in all, the changes made to our ECE program outcomes and objectives, and the resulting enhancements to their assessment, to incorporate the Global Learning Outcomes should result in a stronger learning experience for our students. This may translate into better job opportunities for our more globally-oriented ECE students and potentially serve as an advantage in student recruitment to our program. Several regional employers and industry alumni expressed strong support of our integration of global learning into the ECE curriculum upon learning of our work. 


\section{References}

[1] American Association of Colleges and Universities, http://www.aacu.org/.

[2] Accreditation Board for Engineering and Technology, Inc., http://www . abet.org/.

[3] Educating Americans for a World of Flux: Ten Ground Rules for Internationalizing Higher Education, American Council on Education, Washington, DC, 1995.

[4] IEEE Global History Network, http://www. ieeeghn.org/wiki/index.php/Special:Home

[5] Society for the History of Technology, http://www.historyoftechnology.org/

[6] Under the Economic Turmoil a Skills Gap Simmers, CERI Research Brief 1-2010, February, 2010.

[7] Downey, Gary Lee; Lucena, Juan C.; Moskal, Barbara M.; Bigley, Thomas; Hays, Chris; Jesiek, Brent K.; Kelly, Liam; Lehr, Jane L.; Miller, Jonson; Nichols-Belo, Amy; Ruff, Sharon and Parkhurst, Rosamond, The Globally Competent Engineer: Working Effectively with People Who Define Problems Differently., Journal of Engineering Education, Vol. 95, No. 2, 2006, pp. 107-122.

[8] Elrod, E. and Hovland, K. Global Learning and Scientific Literacy at the Crossroads., Diversity \& Democracy, Vol. 14, No. 2, 2011, pp. 1-4.

[9] Green, M. F.; Luu, D. and Burris, B., Mapping internationalization on U.S. Campuses: 2008 edition, Washington, DC: American Council on Education, 2008.

[10] Nair, Indira, New Scientific Literacies for an Interdependent World, Diversity \& Democracy, Vol. 14, No.2, 2011, pp. 5-7.

[11] Olson, C. L.; Green, M. F. and Hill, B. A., A Handbook for Advancing Comprehensive Internationalization: What Institutions Can Do and What Students Should Learn, Washington, DC: American Council on Education, 2006.

[12] Parkinson, A.; Harb, J. and Magleby, S, Developing Global Competence in Engineers,: What does it mean? What is most important?, Paper 2009-571, Proceedings, 2009 ASEE Annual Conference and Exposition, June 2009. 